\title{
Analysis of the Changes in Processes Using the Kosinski's Fuzzy Numbers
}

\author{
Piotr Prokopowicz \\ Kazimierz Wielki University \\ in Bydgoszcz \\ ul. Chodkiewicza 30, 85-064 Bydgoszcz, Poland \\ Email: piotrekp@ukw.edu.pl
}

\begin{abstract}
This paper presents the analysis of potential of the Kosinski's Fuzzy Number (KFN) idea in the modeling trends of the processes which are described imprecisely. KFNs conception is an alternative for the classical fuzzy numbers ideas as model to represent of the imprecise quantitative data. They introduces new feature into vagueness of the information - a direction. It is base for good arithmetical properties of calculations. Furthermore, a direction also extends a potential in the modeling of information by the additional interpretation, what is a subject of this article. This new potential is presented and explained basing on the example of modeling of quantity of liquid in a reservoir. The environment is changing dynamically what is described as the changes in inflow and outflow. Proposed example explains how to interpret the direction of $\mathrm{KFN}$ and how to understand the results of calculations and its influence.
\end{abstract}

Index Terms-Ordered Fuzzy Numbers, Kosinski's Fuzzy Numbers, direction of imprecision, trend in the data, interpretation of fuzziness

\section{INTRODUCTION}

$\mathbf{T}$ HE CLASSIC idea of fuzzy sets and numbers is widely known methodology for modeling the imprecision of real world. It wins with statistical methods, especially in the problems where partial imprecision is needed.

Unfortunately the computational properties of classical fuzzy numbers (convex fuzzy numbers) have drawbacks connected with a rapidly growing imprecision after sequence of operations. To improve their arithmetics several additional solutions were introduced. They are usually connected with defining additional operations or constraints ([1][2][3]).

An alternative solution is the Kosinski's Fuzzy Numbers mathematical model [4], [5]. This model takes into account the order of the characteristic parts of a fuzzy number giving the fuzzy number an additional feature - direction. By the consideration of the order in calculations we get the opportunity to reduce the imprecision of the following operations. The KFN computational model have a number of properties which were presented in the publications [4], [6], [7].

This model was previously called 'Ordered Fuzzy Numbers'. However, to honor the late Professor Witold Kosiński, his work, the contribution and commitment to the development, analysis and popularization of this model since the 2015 [8] the name "Kosinski's Fuzzy Numbers" (KFN for short) is used instead.
New property - a direction - apart good properties in calculations also introduces new interpretative potential. We have additional information in processing imprecise data associated with the direction. The papers [9], [10] presents a practical use of KFNs in the modeling of financial data, and [11] applies them to the modeling of diversity of opinions in the social networks. Furthermore, in [12] is presented proposition of the application of KFNs for the ant colony optimization algorithm.

A direction is property which can contain an additional portion of information in the same object which also represents an imprecise fuzzy value. However, the interpretation of this new feature, should be consistent and intuitive with methods of the calculations/processing and also with the final results of them. Such coherent proposal how to understand a direction of KFN was presented in [13], [14]. This proposition connect this new feature with a representation of trend in a process. Despite the fact that in the literature can be found various proposals of applications of the model KFN, it lacks exact discuss and full consistent example how and where to get the direction and how it translates into results of operations. Thus the purpose of this publication is to provide such complete analysis of the quite simple and easy to understand process where actions are described by KFNs.

It will be presented on the example of process of changes in the level of liquid in the reservoir. Example presents a problem, in which we want to describe / determine the state of the liquid in the container, under conditions of high variability due to rapidly changing an inflow and an outflow. This publication demonstrates the effectiveness of KFNs in the modeling such problems. The example will show that by modeling the process with the KFNs, we can get more than imprecise description of situation, we can get also the informations about the trends of changes.

\section{A. The Organization of the Paper}

In next section the Kosinski's Fuzzy Number model is shortly introduced with the genesis of the idea. In subsections are also presented the definitions of arithmetic operations on KFNs and practical interpretation of the specific feature of the model - the direction. The following - third section contains the description of the process, which is modeled in this paper. First subsection presents assumptions of the model, next the 


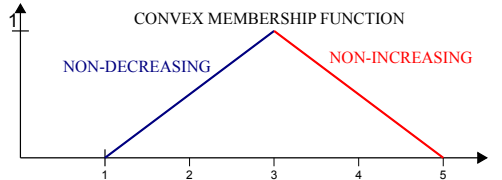

Fig. 1. Parts of the convex membership function

description of subsequent actions with use of KFN model. Third subsection presents the analysis of the appropriateness of using KFNs for modeling of actions of dynamic process. Next - fourth section provides some discussion about different ways for concerning of the direction in the model of process. Final section provides extended summary and conclusions for this paper.

\section{KOSINSKI's FUZZY NUMBER (KFN)}

Main concepts of the idea of Kosinski's Fuzzy Numbers were introduced and developed in the series of papers [15], [4], [5], [6], [13], [16], [14]. As it is an unusual model of imprecise information, before introducing a formal definition, it will be useful to clarify a background of concept.

At the beginning, very important fact must be emphasized. The KFNs are not the fuzzy sets. They are connected conceptually with the idea of fuzzy sets and they can be used in similar way, but, in formal, they are not Zadeh's fuzzy sets. Thus comparing the operations on KFNs with adequate operations based on Zadeh's extension principle [17] can be realized only in the context of the results of calculations, but not by the definitions.

It is also worth noting the fact, although KFNs by definition are other mathematical objects than fuzzy sets, they can represent the majority of situations, which can be described by the convex fuzzy numbers including calculations on them

\section{A. Genesis of the KFNs}

Idea of KFNs have a source in the quasi-concavity of membership functions of fuzzy numbers [18]. It is not a new observation that the each convex membership function of fuzzy number can be split into two parts: first is nondecreasing and second is non-increasing (see fig.1). It is a base of widely known classical fuzzy numbers model called the $(L, R)$ fuzzy numbers [19]. The KFNs idea also generally is based on such point of view. However, the new model treat separately non-decreasing and non-increasing parts of fuzzy number. Additionally, it defines an order between these parts as independent from the domain values. Such conditions leads to the new possibilities in calculations and also in processing of imprecise data.

\section{B. Definition of the $K F N$}

Following the papers [15], [4], [5], [6], [13], [20], [8], [21] fuzzy number will be identified with the pair of functions defined on the interval $[0,1]$.
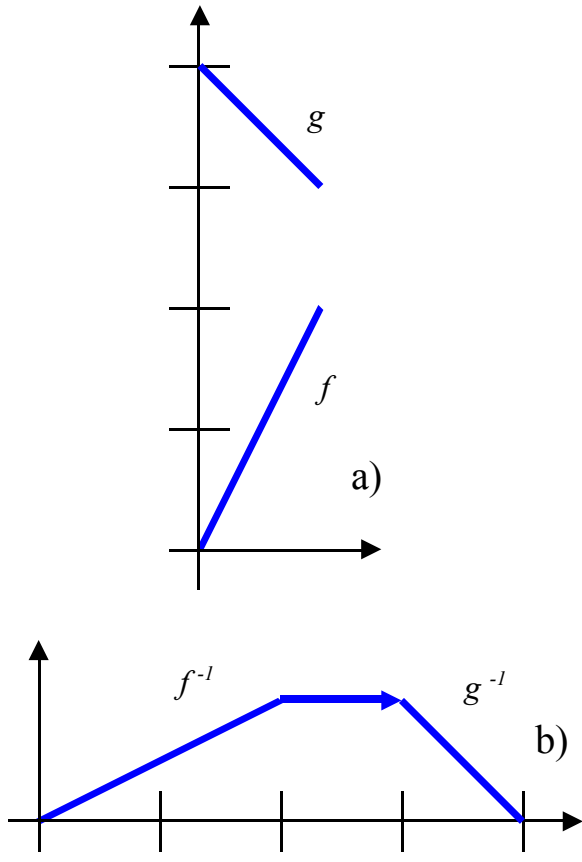

Fig. 2. a)Kosinski's Fuzzy Number, b)The Kosinski's Fuzzy Number as convex fuzzy number with an arrow.

Definition 1: The Kosinski's Fuzzy Number (KFN in short) $A$ is an ordered pair of two continuous functions

$$
A=\left(f_{A}, g_{A}\right)
$$

called the up-part and the down-part, respectively. Both are defined on the closed interval $[0,1]$ with values in $\mathbf{R}$.

If the functions $f$ and $g$ are monotonic (Fig.2a), they are also invertible and possess the corresponding inverse functions defined on the real axis with the values in interval $[0,1]$. If these two inversed functions are not connected, we linking them with constant function with the value 1 . In such way we receive an object which directly represents the classical fuzzy number. For the finalization of transformation, we need to mark an order of $f$ and $g$ with an arrow on the graph (see Fig.2b). Notice that pairs $(f, g)$ and $(g, f)$ are the two different Kosinski's Fuzzy Numbers, unless $f=g$. They differ by their orientation or direction. We can distinguish two orientations giving them names. If the down-part $g$ is greater than the uppart $f$ we will call that "positive" orientation and opposite to it - "negative".

For the later use it will be more convenient to adopt the following general indications of the KFN boundaries:

$$
\begin{aligned}
U P & =\left(s, 1^{-}\right) \\
C O N S T & =\left[1^{-}, 1^{+}\right] \\
D O W N & =\left(1^{+}, e\right)
\end{aligned}
$$

These boundaries allow for simply representation of the KFNs where $f$ and $g$ (up-part and down-part) are linear functions. In such situation we can precisely represent a given KFN by four $\left(s, 1^{-}, 1^{+}, e\right)$. In fact in this paper in the example presented in 
later sections the CONST interval will be minimized to the one point $k=1^{-}=1^{+}$, thus the KFNs will be represented by triples $(s, k, e)$ (see fig.3).

It should be emphasized that, these intervals can be improper intervals (see the KFNs B and C on the fig.3) in the sense of Kaucher's extended interval arithmetic [22] and called by him 'directed intervals', i.e. such $[a, b]$ where $a$ may be greater than $b$.

For monotonous $f$ and $g$ we may point the membership function within the meaning of classical fuzzy numbers:

$$
\mu(x)=\left\{\begin{array}{l}
f^{-1}(x), \text { if } x \in[f(0), f(1)]=\left[s, 1^{-}\right], \\
g^{-1}(x), \text { if } x \in[g(1), g(0)]=\left[1^{+}, e\right], \\
1 \text { when } x \in\left[1^{-}, 1^{+}\right] .
\end{array}\right.
$$

It is worth to point out that a class of Kosinski's Fuzzy Numbers represents the very wide class of convex fuzzy numbers with continuous membership functions (regarding "classical fuzzy numbers" [18], [23], [3], [24]).

\section{Arithmetic Operations}

Operations on KFNs we define as calculations with the upparts and down-parts as follows:

Definition 2: Let $A=\left(f_{A}, g_{A}\right), B=\left(f_{B}, g_{B}\right)$ and $C=$ $\left(f_{C}, g_{C}\right)$ are mathematical objects called Kosinski's Fuzzy Numbers. The sum $C=A+B$, subtraction $C=A-B$, product $C=A \cdot B$, and division $C=A \div B$ are defined by the formula

$$
f_{C}(y)=f_{A}(y) \star f_{B}(y) \wedge \quad g_{C}(y)=g_{A}(y) \star g_{B}(y)
$$

"^" replaces "+", "-", ".", and "/". The $A / B$ is determined only if $B$ does not contain zero. The $y \in[0,1]$ is the domain of functions $f$ and $g$.

The properties of these operations and their results have already been discussed and analyzed in various publications (for example see [25], [6], [7]). Additionally the paper [7] presents also a different examples of calculations. Nevertheless a brief description of the properties KFN calculations is needed for a better understanding of analysis included in the next sections of this paper. For example it is important property, that the subtraction is equal to the addition of the opposite number, where the opposite number is obtained by multiplying the given value by the -1 (real number - singleton). By using the above-mentioned method in calculation of $A-A$ we obtain exact zero (crisp number). Using the arithmetical operations on KFNs every simple equation type $A+X=B$, where $A$ and $B$ are fuzzy numbers with any membership functions, can be solved. We calculate result exactly the same way as with real numbers. Such possibilities are a consequence of adopting operations for KFNs directly from real numbers. After a closer investigation of the definitions 1 and 2 it can be noted that the operations on parts of the KFNs are executed through operations on functions representing these parts. Finally, the operations on the functions are, in fact, operations on their values. Thus, if space of values of function

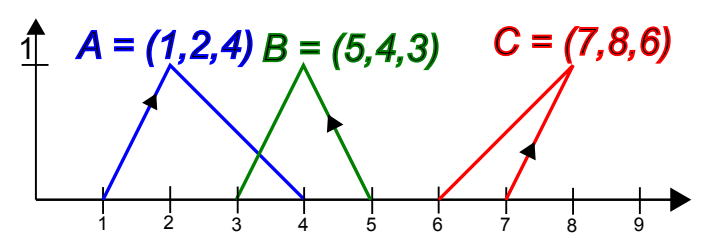

Fig. 3. Examples of KFNs represented by triples.

are the real numbers (as with all KFNs), then practically, calculations are executed as operations on the real numbers universe. Summarizing, the KFN model grants flexibility of calculating on imprecise data in a similar way like with real numbers on crisp data. It retains fuzzy quantitative character, but without necessity of growing an imprecision.

However, using the KFNs we should remember that their basics are different than the Zadeh's fuzzy sets and some objects like $C$ on the fig.3 can appear (see also [14], [7]). Such elements are consistent with the definition of KFN (see def.1), although, their shape can not be defined as a membership function. Such objects are called improper KFNs. This aspect of model was commented in [4], [5], [7]. Despite the unusual shape (as for fuzzy numbers), such KFNs still contain important information needed for the calculations. In the example presented in next sections of this paper such object also will be part of analysis as an important portion of data about the modeled process.

\section{Imprecision interpretation and direction of the KFN}

The direction is a key element of the KFN model. Basically it is defined as an order of the parts of fuzzy number and is independent from the real numbers universe. Proposition of practical interpretation for this new property was presented in [13], [14]. KFNs are considered in these papers as the values representing an observation, which passes in time, regardless of the order of numerical values. So, the time dependence can be a natural interpretation of the direction. Such context is also used in this publication. Following [13], [14]imprecision here is interpreted as a consequence of dynamic changes. So the up-part represents, relatively short, past behavior of the value represented by given $\mathrm{KFN}$, and the down-part indicate expected change of the value in the next step of process. However, it should be clarified that this is not the only possible interpretation of the direction, but this publication focuses just on such variant.

\section{MOdeling PROCESS USING THE KFNS}

At start it should be noted, that there is a paper [26] that presents an example of calculations which context is connected with the water lever for the cofferdam. But that publication focuses on comparison of arithmetics of LR fuzzy numbers and KFNs based on solving simple equation. The issue of the direction's source is there minimized.

Possibilities of modeling an imprecision in the processes with use of the KFNs will be presented here on the simple intuitive example of reservoir with one outflow and one inflow. 


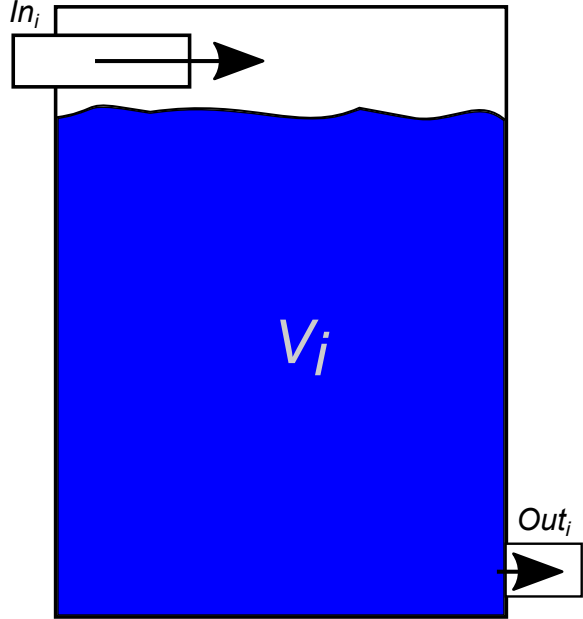

Fig. 4. General idea of the model.

KFNs are used here to describe a behavior of the inflow and the outflow, and also, to indicate the amount of liquid in the reservoir during each step of the process. Following [13], [14], here, the source of imprecision will be also connected strictly with a dynamic changes of parameters. Thus, when no changes are applied the situation will be considered as crisp and precise.

It is worth noting that the problem that we want to solve with this example is to find a simple method to determine the current state of the liquid in the tank in a high variability situation. The input data, in addition to the initial state, are the changes in inflow and outflow. Generally, the problem can be formulated as monitoring the status of the tank only on the basis of the monitoring of sources (an outflow is interpreted as a negative value).

\section{A. Assumptions}

General idea of the model is presented on the fig. 4. Before the analysis, some assumptions should be clarified. The time of analyzed example is discrete and divided onto intervals with a similar duration. The units are not specified, as it is not relevant whether they will be seconds, minutes or hours. The actions during the process will be described as changes of values (of inflow and outflow) which lasts by one time interval denoted by $\Delta t$. Similarly, the determination of the state of actual liquid level in the reservoir will be denoted by $V_{i}$ without specifying units. As the natural consequence of these assumptions the performance of inflow $I n_{i}$ and outflow $O u t_{i}$ will be described as cumulative change $\Delta V_{i}$ during $\Delta t$ with units adequate to the description of capacity of analyzed reservoir and timesteps. The index $i$ will indicate the subsequent time-steps in the analysis.

\section{B. The sequence of actions in the process}

The exemplary of process starts with static situation. Inflow and outflow is zero. At the beginning, volume of liquid in the reservoir is described by the KFN singleton $V_{0}=(20,20,20)$.
The whole process will be divided onto time-steps numerated from 1 to 11 . Each step is described by the changes in inflow and outflow which are usually consequent continuations of previous states. For each step, the actual volume of liquid will be calculated by the formula:

$$
V_{i}=V_{i-1}+\operatorname{In}_{i}+O u t_{i}
$$

where $i$ - indicates the number of subsequent - actually analyzed - time interval. In general, the inflow values are positive numbers as they represent incoming liquid to the reservoir. So, for the outflow the values are negative.

Steps in the process are as follows:

1) The inflow starts slowly from 0 to 1 . It is described by the $\mathrm{KFN} \operatorname{In}_{1}=(0,0,1)$. The outflow is no changing so the value is Out $_{1}=(0,0,0)$. The state of liquid in the reservoir is calculated $V_{1}=V_{i-1}+I n_{i}+O u t_{i}=$ $(20,20,21)$. As we can see the fuzziness is growing due to the changes in inflow. The result represents not only the actual state but also an information about a trend in the process (which is presently growing due to positive orientation of $V_{1}$ ).

2) The inflow is growing with the same speed $\mathrm{In}_{2}=$ $(0,1,2)$. It means is increasing from 1 to 2 unit of volume in $\Delta t$. The outflow still is closed: $O_{u} t_{2}=$ $(0,0,0)$. The level of liquid: $V_{2}=V_{i-1}+I n_{i}+O u t_{i}=$ $(20,21,23)$. We can see that we again have reasonable imprecise information about actual volume of liquid. Summarizing, it started with 20 then in first time interval the inflow was about 0 however not precisely. During next time interval, the inflow is growing and is about 1 . So after these two time intervals we have in result the content of reservoir about 21 . The orientation is positive so this is increasing trend and as we can see, generally, it is the right conclusion for this moment.

3) The inflow is growing with the same speed $\mathrm{In}_{3}=$ $(1,2,3)$. Now the outflow is activated and it is rapid: Out $_{3}=(0,0,-3)$. At this point it is worth to calculate the cumulative change: $\Delta V_{3}=I_{3}+O u t_{3}=(1,2,0)$. The level of liquid: $V_{3}=(21,23,23)$. In this step we see, that the starting rapid outflow eliminates the effect of inflow. The KFN $V_{3}$ tells us that the growing trend in filling reservoir is stopping. More interesting however, is the cumulative change $\Delta V_{3}$, which is in fact the improper KFN as it was earlier introduced. As we can see such object represents important information sudden change of a trend.

4) The inflow is growing with the same speed: $I n_{4}=$ $(2,3,4)$. The outflow is growing with the same high speed: Out $_{4}=(0,-3,-6)$. The cumulative change: $\Delta V_{4}=I_{4}+$ Out $_{4}=(2,0,-2)$. The level of liquid: $V_{4}=(23,23,21)$. In this step we have a situation when the actual volume of content of reservoir changes orientation. Inflow and outflow are more or less balancing, however trend starts decreasing.

5) The inflow stops increasing at the level 4: $I n_{5}=$ $(3,4,4)$. The outflow still is growing, however, much 

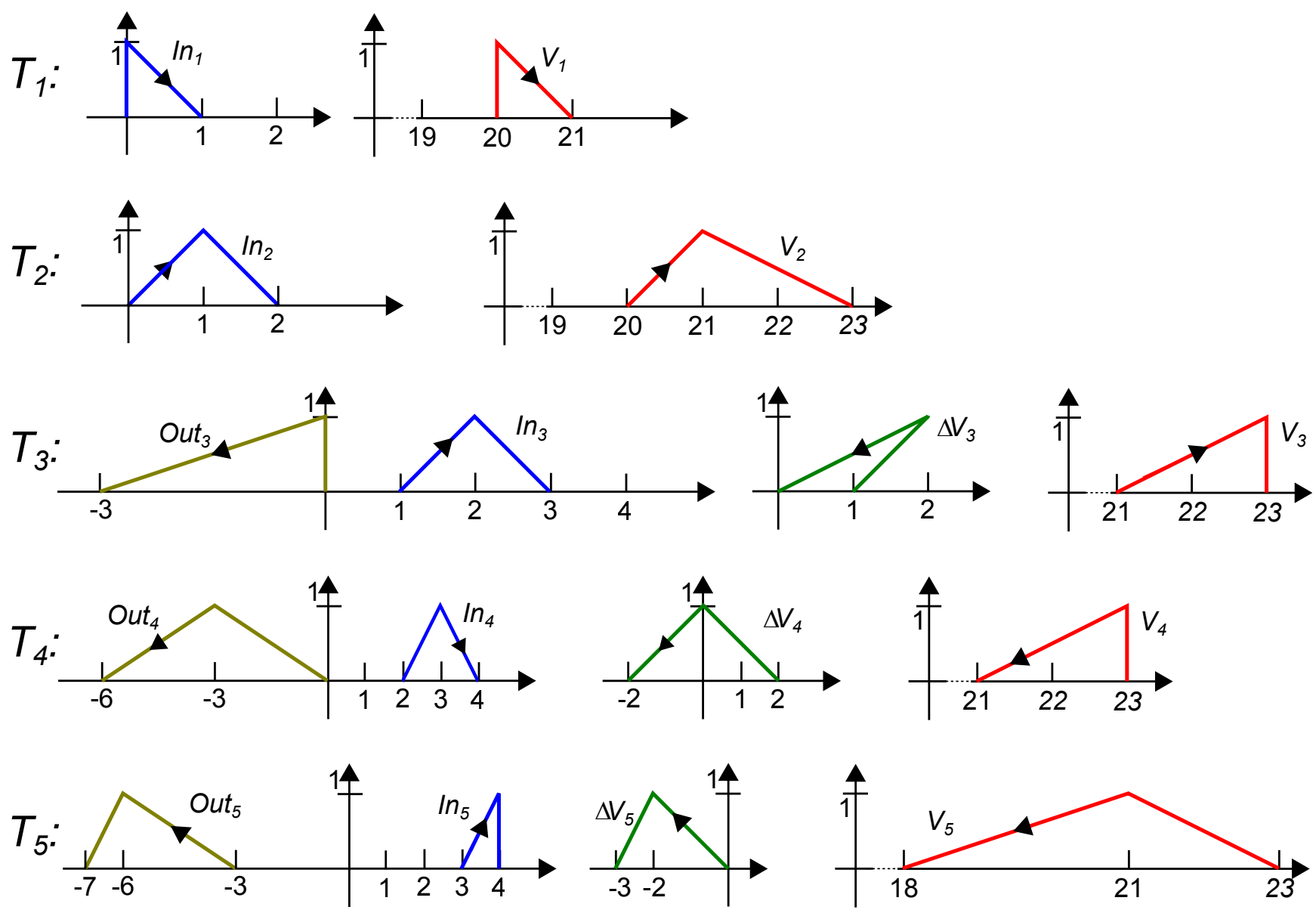

Fig. 5. KFNs in first five time intervals of the process.

more slowly than before: Out $_{5}=(-3,-6,-7)$. The cumulative change: $\Delta V_{5}=(0,-2,-3)$. The level of liquid: $V_{5}=(23,21,18)$. Clearly as the $V_{5}$ posses negative orientation the liquid volume is about 21 in the decreasing trend.

6) The inflow is constant and equal 4: $\operatorname{In}_{6}=$ $(4,4,4)$. The outflow stops growing at the value $-7:$ Out $_{6}=(-6,-7,-7)$. The cumulative change: $\Delta V_{6}=(-2,-3,-3)$. The actual level of liquid: $V_{6}=$ $(21,18,15)$. At this moment we have a situation where the outflow and the inflow stabilizing. So, fuzziness of cumulative change is not high, however, the imprecision of actual volume of liquid is clearly greater. Although the cumulative changes are not too large, the situation in the reservoir is quite dynamic. In comparison with the initial value of 20 , currently we have an income of liquid on the level 4 and outcome - about 6 . This is generally quite big change, even if cumulatively it is not so significant.

7) The inflow starts decreasing with high dynamic: $\mathrm{In}_{7}=(4,4,2)$. The outflow is stabilized at -7 : Out $_{7}=(-7,-7,-7)$. The cumulative change: $\Delta V_{7}=$ $(-3,-3,-5)$. The level of liquid $V_{7}=(18,15,10)$.
Now we have clear decreasing trend and this decreasing is growing.

8) The inflow decreasing with the same speed: $I n_{8}=$ $(4,2,0)$. The outflow starts rapidly decreasing: $O u t_{8}=$ $(-7,-7,-4)$. The cumulative change: $\Delta V_{8}=$ $(-3,-5,-4)$. The level of liquid: $V_{8}=(15,10,6)$. Once again we have to deal with the improper KFN as cumulative change.

9) The inflow is closing: $I n_{9}=(2,0,0)$. The outflow is decreasing with the same very high speed: Out $_{9}=(-7,-4,-1)$. The cumulative change: $\Delta V_{9}=$ $(-5,-4,-1)$. The level of liquid: $V_{9}=(10,6,5)$.

10) The inflow is cut off: $I n_{10}=(0,0,0)$. The outflow is closing: Out $t_{10}=(-4,-1,0)$. The level of liquid: $V_{10}=(6,5,5)$. This KFN shows that situation in reservoir is stabilizing. The whole process stops, so a fuzziness/imprecision is low.

11) Finally, the inflow is cut off and also the outflow definitely ends: Out $_{11}=(-1,0,0)$. The final level of liquid: $V_{11}=(5,5,5)$. There is no change in the reservoir. Therefore, according to previous assumptions (a source of imprecision are changes), the result is not fuzzy. 


\section{The KFNs in the example}

For a better understanding and analysis of the example the full graphical representation of KFNs which describes clue values for first five steps of the process are presented on fig.5. Summing up, we can identify the following general activities during the process:

- First, the inflow is slowly turning on and it is increasing steadily giving more and more income of the liquid during next few time intervals. After that it stabilizes for a moment.

- During slow increasing of inflow, the rapid outflow starts, which quickly slow down and stabilizes in short time.

- Then, the inflow is slowly closing and then, almost simultaneously, also the outflow is closing.

The KFN model can be represented, quite intuitively, by Japanese Candlestick Chart commonly used for financial data [10]. We can use it to present a set of KFNs which have a common context. This kind of chart is especially compatible with the KFNs because it can represent not only values, but also intervals and more important - a direction. It is not full precise description but gives general outlook about main character of such set. Figure 6 represents the chart for volume of liquid $V_{i}$ in the reservoir during each time interval. The actual level of content during $i-t h$ time interval is represented by the rectangles. Height of the rectangle represents the range of imprecision. Additionally, white color represents the increasing trend and black - the decreasing. So, we can read from the chart informations about the content of reservoir during subsequent time-steps as follows:

- The trend is positive in the first three time intervals. The fuzziness is not large as the changes are not so dynamic.

- From the fourth time-step the trend changes and becomes negative. Additionally the fuzziness is growing. If we refer back to the detailed description of the example, in third step the outflow starts rapidly and lasts for a while. So, its consequence is the change of trend in fourth step and growing imprecision during next few periods.

- From the ninth time interval, imprecision is decreasing, however trend of the liquid's volume is still negative. If we again refer back to the detailed description of the process, we notice that from ninth period the dynamics of inflow and outflow is decreasing so the imprecision is smaller.

\section{DETERMINING DIRECTION OF THE KFNS}

The source of a direction of KFNs used in the example is basing on the fundamentals of conception 'a change', which has generally two possible trends. It is either increasing or decreasing - thus positive or negative orientation for the KFNs. It is simple and intuitive.

In the linguistic description of actions in the example from previous section, we consider the inversion of ideas: the inflow and the outflow. Therefore, if the inflow means adding liquid to the reservoir, then enlarging an outflow means that, the KFN becoming more and more negative. The KFN model grants

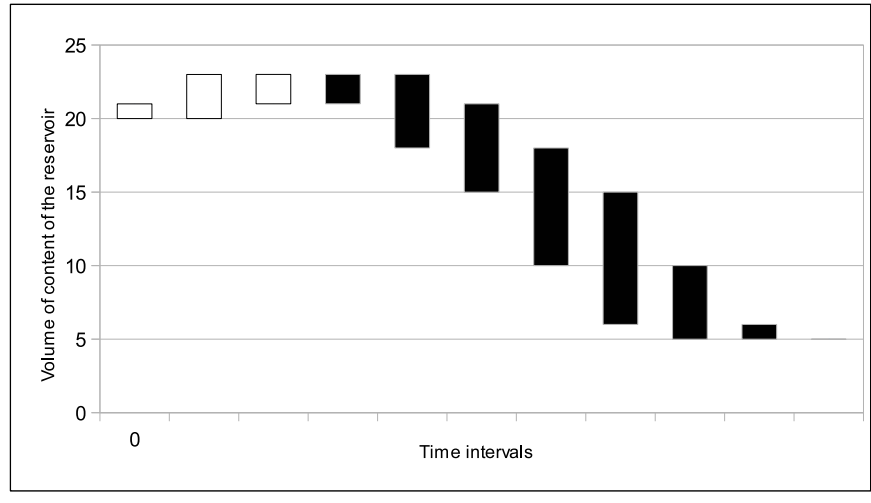

Fig. 6. Candlestick chart for the level of liquid in the subsequent time-steps.

flexibility of calculations, so we could describe outflow with absolute values. However, in such situation the opposition of outflow against inflow should be referred in the calculating of actual volume of content. Instead the formula 5 we should use:

$$
V_{i}=V_{i-1}+I n_{i}-O u t_{i} .
$$

Finally, the results would not change, due to the fact that subtracting the KFNs is, in real, adding the opposite number. We get the opposite number like in the real numbers (but unlike with classical fuzzy numbers) by multiplying the original number by -1 .

\section{SUMmARY AND CONCLUSIONS}

The example in the previous section presents application of KFNs for the modeling of changes in the process of filling and outpouring content in the reservoir. Although this example is simply, it presents not only the changes of actual volume of liquid. In fact, the fuzziness of inflow and outflow refers to the 'change of changes'. As the $I n_{i}$ and $O u t_{i}$ represents $\Delta V_{i} / \Delta t$, so fuzziness of them is a change $\Delta\left(\Delta V_{i}\right)$ during $\Delta t$. It may be easier to understand if we compare this with the process of object's movement. If $x$ is a position, then $\Delta x$ is a velocity. And furthermore, change of speed is an acceleration. It generally shows a good potential of the KFNs as a good tool for modeling more complex dynamic action than just simple changes.

Presented process is analyzed step by step. It shows also particular usefulness of KFN model for a linguistic describing of changes. Although in the example we have one inflow and outflow, it is not difficult to expand conception to the multiinflow/outflow situations. The formula 5 becomes as follows:

$$
V_{i}=V_{i-1}+\Sigma I n_{i}^{j}+\Sigma O u t_{i}^{k}
$$

where: $j$ - subsequent number of the inflow, $k$ - subsequent number of the outflow.

However, because the indication of source of changes, if it is either inflow or inflow, bases on the numerical values, we can simplify the notation. Instead $I n^{j}$ and $O u t^{k}$ we can use just $S r c$ :

$$
V_{i}=V_{i-1}+\Sigma S r c_{i}^{m}
$$


where: $m \in(j+k)$ - subsequent number of the source of change of any kind.

If we analyze concrete values of actual level of liquid $V_{i}$ in each time-step (let's look more closely at the fig.5) we can notice that KFNs accumulates the fuzziness of changes. However, if changes are opposite - they are canceling. This is right and consistent with the intuition. If the liquid is inflowing and simultaneously the same amount of content is outflowing, there is no change at all.

Furthermore we can see also on fig. 5, that KFNs representing actual volume of content can be referred to the classical fuzzy numbers. Such objects can be defuzzified or we can ignore a direction and use them, for example, as input values for the next stage of processing, even with use of classical fuzzy system. But these KFNs contains more information than only imprecision. We can read from them, the actual trend of the process. The papers [16], [27], [8], [21] describes the research of the methods of processing of KFNs where a direction is considered - Direction Sensitive Fuzzy Information Processing. So, instead ignoring orientation we can use full information and process it in a more effective way.

By application of KFNs we can distinguish a situation "about 5 in increasing trend" and "about 5 in decreasing trend". It is not hard to find many real life circumstances where these informations should generate significantly different reactions. An example can be the getting dressed for a trip. If we have "15 degrees of Celsius and temperature is decreasing" we should get more warm cloth than in opposite trend. More to that, there is also significance either a dynamic of changes is large or small.

The example presented in this paper can be easy transferred into other areas than the controlling content of reservoir. Balancing of the incomes and outcomes is very popular pattern. If it is inflow of liquid or income of money, or increasing number of some web-portal users, it can be described by the KFNs. So, if we need a tool for processing an imprecise data and we want to model a trend, the KFNs can be used.

ON closing, it should be stressed that, this publication presents the effectiveness of the use of KFNs in the situations where we focus on the dynamic changes as the source of the inaccuracy. However, there is many cases where a vagueness is more complex. In such situations a good idea is to design the hybrid methods which will consider all significant sources of an imprecision.

\section{REFERENCES}

[1] E. Sanchez, "Solution of fuzzy equations with extended operations," Fuzzy Sets and Systems, vol. 12, no. 3, pp. 237 - 248, 1984. [Online]. Available: http://dx.doi.org/10.1016/0165-0114(84)90071-X

[2] G. J. Klir, "Fuzzy arithmetic with requisite constraints," Fuzzy Sets and Systems, vol. 91, no. 2, pp. 165 - 175, 1997, fuzzy Arithmetic. [Online]. Available: http://dx.doi.org/10.1016/S0165-0114(97)00138-3

[3] M. Wagenknecht, R. Hampel, and V. Schneider, "Computational aspects of fuzzy arithmetics based on archimedean t-norms," Fuzzy Sets and Systems, vol. 123, no. 1, pp. 49 - 62, 2001. [Online]. Available: http://dx.doi.org/10.1016/S0165-0114(00)00096-8

[4] W. Kosiński, P. Prokopowicz, and D. Slezzak, "Ordered fuzzy numbers," Biulletin of the Polish Academy of Sciences Mathematics, vol. 51, no. 3 , pp. $327-338,2003$
[5] W. Kosiński, P. Prokopowicz, and D. Ślezak, Intelligent Information Processing and Web Mining: Proceedings of the International IIS IIPWM'03 Conference held in Zakopane, Poland, June 2-5, 2003. Berlin, Heidelberg: Springer Berlin Heidelberg, 2003, ch. On Algebraic Operations on Fuzzy Numbers, pp. 353-362. [Online]. Available: http://dx.doi.org/10.1007/978-3-540-36562-4 37

[6] — "Calculus with fuzzy numbers," in Intelligent Media Technology for Communicative Intelligence, ser. Lecture Notes in Compute Science, L. Bolc, Z. Michalewicz, and T. Nishida, Eds. Springe Berlin Heidelberg, 2005, vol. 3490, pp. 21-28. [Online]. Available: http://dx.doi.org/10.1007/11558637_3

[7] P. Prokopowicz, "Flexible and simple methods of calculation on fuzzy numbers with the ordered fuzzy numbers model," in Artificial Intelligence and Soft Computing, ser. Lecture Notes in Computer Science, L. Rutkowski, M. Korytkowski, R. Scherer, R. Tadeusiewicz, L. Zadeh, and J. Zurada, Eds. Springer Berlin Heidelberg, 2013, vol. 7894, pp. 365-375. [Online]. Available: http://dx.doi.org/10.1007/978-3-642-38658-9_33

[8] P. Prokopowicz and W. Pedrycz, "The directed compatibility between ordered fuzzy numbers - a base tool for a direction sensitive fuzzy information processing," in Artificial Intelligence and Soft Computing, ser. Lecture Notes in Computer Science, L. Rutkowski, M. Korytkowski, R. Scherer, R. Tadeusiewicz, L. A. Zadeh, and J. M. Zurada, Eds Springer International Publishing, 2015, vol. 9119, pp. 249-259. [Online]. Available: http://dx.doi.org/10.1007/978-3-319-19324-3_23

[9] A. Marszałek and T. Burczyński, "Modelling financial high frequency data using ordered fuzzy numbers," in Artificial Intelligence and Soft Computing, ser. Lecture Notes in Computer Science, L. Rutkowski, M. Korytkowski, R. Scherer, R. Tadeusiewicz, L. Zadeh, and J. Zurada, Eds. Springer Berlin Heidelberg, 2013, vol. 7894, pp. 345-352. [Online]. Available: http://dx.doi.org/10.1007/978-3-642-38658-9 31

[10] D. Kacprzak, W. Kosiński, and K. W. Kosiński, Artificial Intelligence and Soft Computing: 12th International Conference, ICAISC 2013, Zakopane, Poland, June 9-13, 2013, Proceedings, Part I. Berlin, Heidelberg: Springer Berlin Heidelberg, 2013, ch. Financial Stock Data and Ordered Fuzzy Numbers, pp. 259-270. [Online]. Available: http://dx.doi.org/10.1007/978-3-642-38658-9_24

[11] M. Kacprzak, W. Kosiński, and K. Węgrzyn-Wolska, Artificial Intelligence and Soft Computing: 12th International Conference, ICAISC 2013, Zakopane, Poland, June 9-13, 2013, Proceedings, Part I. Berlin, Heidelberg: Springer Berlin Heidelberg, 2013, ch. Diversity of Opinion Evaluated by Ordered Fuzzy Numbers, pp. 271-281. [Online]. Available: http://dx.doi.org/10.1007/978-3-642-38658-9_25

[12] J. M. Czerniak, Ł. Apiecionek, and H. Zarzycki, Beyond Databases, Architectures, and Structures: 10th International Conference, BDAS 2014, Ustron, Poland, May 27-30, 2014. Proceedings. Cham: Springer International Publishing, 2014, ch. Application of Ordered Fuzzy Numbers in a New OFNAnt Algorithm Based on Ant Colony Optimization, pp. 259-270. [Online]. Available: http://dx.doi.org/10. 1007/978-3-319-06932-6_25

[13] W. Kosinski and P. Prokopowicz, "Fuzziness - representation of dynamic changes?" in New Dimensions in Fuzzy Logic and Related Technologies, Vol. I, Proceedings, M. Stepnicka, V. Novak, and U. Bodenhofer Eds. European Soc Fuzzy Log \& Technol, 2007, pp. 449-456, 5th Conference of the European-Society-for-Fuzzy-Logicand-Technology, Ostrava, Czech Republic, Sep. 11-14, 2007.

[14] W. Kosiński, P. Prokopowicz, and D. Kacprzak, Views on Fuzzy Sets and Systems from Different Perspectives: Philosophy and Logic, Criticisms and Applications. Berlin, Heidelberg: Springer Berlin Heidelberg, 2009, ch. Fuzziness - Representation of Dynamic Changes by Ordered Fuzzy Numbers, pp. 485-508. [Online]. Available: http://dx.doi.org/10.1007/978-3-540-93802-6_24

[15] W. Kosiński, P. Prokopowicz, and D. Ślezak, Neural Networks and Soft Computing: Proceedings of the Sixth International Conference on Neural Networks and Soft Computing, Zakopane, Poland, June 11-15, 2002. Heidelberg: Physica-Verlag HD, 2003, ch. On Algebraic Operations on Fuzzy Reals, pp. 54-61. [Online]. Available: http://dx.doi.org/10.1007/978-3-7908-1902-1_8

[16] P. Prokopowicz, "Adaptation of rules in the fuzzy control system using the arithmetic of ordered fuzzy numbers," in Artificial Intelligence and Soft Computing - ICAISC 2008, ser. Lecture Notes in Computer Science, L. Rutkowski, R. Tadeusiewicz, L. Zadeh, and J. Zurada, Eds. Springer Berlin Heidelberg, 2008, vol. 5097, pp. 306-316. [Online]. Available: http://dx.doi.org/10.1007/978-3-540-69731-2_30 
[17] L. Zadeh, "The concept of a linguistic variable and its application to approximate reasoning I," Information Sciences, vol. 8, no. 3, pp. $199-$ 249, 1975. [Online]. Available: http://dx.doi.org/10.1016/0020-0255(75) 90036-5

[18] H. T. Nguyen, "A note on the extension principle for fuzzy sets," Journal of Mathematical Analysis and Applications, vol. 64, no. 2 pp. 369 - 380, 1978. [Online]. Available: http://dx.doi.org/10.1016/ $0022-247$ X(78) $90045-8$

[19] D. Dubois and H. Prade, "Operations on fuzzy numbers," International Journal of Systems Science, vol. 9, no. 6, pp. 613-626, 1978, cited By 1218. [Online]. Available: http://dx.doi.org/10.1080/00207727808941724

[20] W. Kosiński, P. Prokopowicz, and A. Rosa, "Defuzzification functionals of ordered fuzzy numbers," Fuzzy Systems, IEEE Transactions on, vol. 21, no. 6, pp. 1163-1169, Dec 2013. [Online]. Available: http://dx.doi.org/10.1109/TFUZZ.2013.2243456

[21] P. Prokopowicz, Proceedings of the Second International Afro-European Conference for Industrial Advancement AECIA 2015. Cham: Springe International Publishing, 2016, ch. The Directed Inference for the Kosinski's Fuzzy Number Model, pp. 493-503. [Online]. Available: http://dx.doi.org/10.1007/978-3-319-29504-6 46

[22] E. Kaucher, Fundamentals of Numerical Computation (Computer Oriented Numerical Analysis). Vienna: Springer Vienna, 1980, ch. Interval Analysis in the Extended Interval Space IR, pp. 33-49.
[Online]. Available: http://dx.doi.org/10.1007/978-3-7091-8577-3_3

[23] W. Pedrycz and F. Gomide, An introduction to fuzzy sets: analysis and design. With a foreword by Lotfi A. Zadeh. Cambridge, MA: MIT Press, 1998.

[24] A. Piegat, Fuzzy modeling and control. Heidelberg: Physica-Verlag, 2001

[25] R. Kolesnik, P. Prokopowicz, and W. Kosinski, "Fuzzy calculator - useful tool for programming with fuzzy algebra," in Artificial Intelligence and Soft Computing - ICAISC 2004, 7th International Conference, Zakopane, Poland, June 7-11, 2004, Proceedings, 2004, pp. 320-325. [Online]. Available: http://dx.doi.org/10.1007/978-3-540-24844-6_45

[26] J. M. Czerniak, W. Dobrosielski, Ł. Apiecionek, and D. Ewald, "Representation of a trend in ofn during fuzzy observance of the water level from the crisis control center," in Proceedings of the 2015 Federated Conference on Computer Science and Information Systems, ser. Annals of Computer Science and Information Systems, M. Ganzha, L. Maciaszek, and M. Paprzycki, Eds., vol. 5. IEEE, 2015, pp. 443-447. [Online]. Available: http://dx.doi.org/10.15439/2015F217

[27] P. Prokopowicz and S. Golsefid, "Aggregation operator for ordered fuzzy numbers concerning the direction," in Artificial Intelligence and Soft Computing, ser. Lecture Notes in Computer Science, L. Rutkowski, M. Korytkowski, R. Scherer, R. Tadeusiewicz, L. Zadeh, and J. Zurada, Eds. Springer International Publishing, 2014, vol. 8467, pp. 267-278. [Online]. Available: http://dx.doi.org/10.1007/978-3-319-07173-2 24 